\title{
Züchtungsexperimente an zwei marinen Flagellaten (Dinophyta) und ihre Anwendung im toxikologischen Abwassertest
}

\author{
H. KAYSER \\ Biologische Anstalt Helgoland, Meeresstation, Helgoland
}

\begin{abstract}
Rearing experiments on two marine flagellates (Dinophyta) and their application to a toxicological waste water test. During rearing experiments with Prorocentrum micans and Ceratium furca (Dinophyta) under different conditions of temperature, light and nutrients, the role of industrial waste water of a titaniumdioxide factory has been examined. The waste water contains primarily $\mathrm{H}_{2} \mathrm{SO}_{4}$ and $\mathrm{FeSO}_{4}$ (for details consult Table 1); it is scheduled for discharge in early 1969 in quantities of some 1,200 tons per day into a North Sea area about 12 nautical miles north-west of Helgoland. The organisms were cultivated in bottles containing limited volumes of test liquid, and in chemostats, over periods of a few weeks. Growth rates of populations exposed to different waste water concentrations served as criterion of possible toxic effects of the industrial waste water. The experiments revealed distinct sublethal effects in dilutions (parts waste water : parts seawater of $32 \% \mathrm{~S}$ ) down to $1: 50,000$ (Ceratium furca), and $1: 32,000$ (Prorocentrum micans). At $1: 5,000$ only a portion of the population (Prorocentrum micans) survives; the remaining cells stagnate reproduction at a given constant cell density. The specific toxic effects of the different components of the waste water are discussed. Further experiments are necessary to increase our knowledge on the biological consequences of the discharge of waste water into the North Sea, and to develop methods for the assessment of the effects of unknown toxic agents by means of culture experiments.
\end{abstract}

\section{EINLEITUNG}

Die Kenntnisse der Biologie mariner Organismen stützen sich auf Beobachtungen in freier Natur und auf die Ergebnisse von Experimenten im Labor. Das Experiment erlaubt, durch bestimmte Versuchsanordnungen gezielte Fragen an das Objekt zu stellen. Seine Reaktion liefert uns die Antwort. Okologische Fragestellungen erfordern eine Versuchsanordnung, die den Bedingungen in freier Natur möglichst entsprechen; erst dann erhält das im Labor gewonnene Versuchsergebnis Aussagekraft für die Reaktion der Organismen im natürlichen Milieu.

Vorliegende Arbeit berichtet über die Ergebnisse experimentell-ökologischer Untersuchungen an zwei Phytoplankton-Organismen, Prorocentrum micans und Ceratium furca (Dinophyta). Ziel der Versuche ist es, zunächst Grundlagen über die Lebensansprïche der untersuchten Arten hinsichtlich Temperatur, Licht und Nährstoffbedarf 
zu erarbeiten. Anhand der auf diese Weise ermittelten optimalen Kulturbedingungen soll ein zweiter Fragenkomplex untersucht werden: der Einfluß toxischer Industrieabwässer.

Dieses iber die Grundlagenforschung hinausgehende zweite Thema aus der angewandten Wissenschaft ergibt sich aus den immer stärker werdenden Bestrebungen der Industrie, ihre Abwässer in das Meer einzubringen. Die Beurteilung der Auswirkungen industrieller Abwässer auf marine Lebensgemeinschaften kann zunächst nur mit Hilfe von Experimenten im Labor durchgefüht werden. Es erscheint daher sinnvoll, im Rahmen experimentell-ökologisch orientierter Zuchtversuche an Phytoplanktonorganismen Toxizitätsuntersuchungen mit industriellen Abwässern durchzuführen.

Toxikologische Versuche im Kulturverfahren bieten die Möglichkeit, subletale Einflüsse über beliebig viele Generationen hinweg zu erfassen. Als Parameter dient die Vermehrungsrate, die sich als wesentlich empfindlicheres Merkmal erweist als die Ermittlung der Sterblichkeit (Dosis letalis). Die Vermehrungsrate wurde auch von BERNHARD, ZATTERA \& Filesi (1966) zur Beurteilung der Toxizität verschiedener Werkstoffe und Detergentien auf pelagische Planktonalgen herangezogen. Das Kulturverfahren erlaubt es, langfristige Experimente im Durchströmungsverfahren (Chemostattechnik) durchzuführen, das den Verhältnissen im Meer weitgehend entspricht, da die Erschöpfung der toxischen Agentien nur sehr langsam oder gar nicht stattfindet (siehe auch HuEcK 1967). Ein weiterer Vorteil des toxikologischen Kulturexperimentes mit Protozoen ist darin zu sehen, daß jeder Einzelversuch an einer ganzen Population durchgeführt wird. Die erhaltenen Aussagen beziehen sich nicht auf Einzelindividuen, sondern erfassen die ganze Reaktionsbreite eines Zuchtstammes.

Kulturexperimente im Labor erfordern zunächst eine Einschränkung in der Wahl der Versuchsorganismen: Robuste und gegen Umwelteinflüsse wenig empfindliche Arten lassen sich gut kultivieren; im toxikologischen Test erweisen sie sich daher als die widerstandsfähigsten Formen.

Arten mit geringerer ökologischer Valenz verlangen hohe Ansprüche in der Kulturtechnik und reagieren im Test empfindlicher. Dem Ókologen muß es daher angelegen sein, eine Auswahl zu treffen, die ihm Aussagen über die ganze Reaktionsbreite eines Okosystems erlaubt und robuste und empfindliche Formen einschließt. Eine weitere Bedingung, die bei der Wahl der Versuchsorganismen berücksichtigt werden sollte, ist ihre Bedeutung im Stoffhaushalt der freien Natur. Arten, die im Meer zu bestimmten Jahreszeiten dominieren, müssen den Vorzug haben vor seltenen oder nur sporadisch auftretenden Formen.

Im Hinblick auf die in dieser Arbeit vorgelegten Versuchsergebnisse an Dinoflagellaten ist Prorocentrum micans als robust und Ceratium furca als empfindlich in der Kultur anzusehen. Beide Arten kommen im Plankton der Nordsee häufig vor, können zeitweise sogar Anlaß zu Wasserblüten geben.

Der unmittelbare Anlaß für die Aufnahme toxikologischer Untersuchungen gab der Antrag der Titangesellschaft m.b.H., Leverkusen, ab Anfang 1969 täglich etwa 1200 Tonnen schwefelsäure- und eisensulfathaltige Abwässer in einem Seegebiet ca. 12 Seemeilen nordwestlich von Helgoland $\left(54^{0} 20^{\prime} \mathrm{N}-54^{0} 25^{\prime} \mathrm{N} ; 7^{0} 35^{\prime} \mathrm{E}-7^{0} 39^{\prime} \mathrm{E}\right) \mathrm{zu}$ verklappen. Über die Konsequenzen, die sich für die Biocoenose dieses und benachbarter Seegebiete ergeben, ist bisher wenig bekannt. Von dem Institut für Küsten- und Bin- 
nenfischerei der Bundesforschungsanstalt für Fischerei in Hamburg wurde ein Gutachten angefertigt. Weitere Untersuchungen wurden von der Biologischen Anstalt Helgoland in Angriff genommen (Kinne \& Rosenthal 1967, Kinne \& Schumann 1968). Die letztgenannten Arbeiten befassen sich mit der Einwirkung des Industrieabwassers auf Jugendstadien von Meeresfischen. In der hier vorgelegten Arbeit wird der Einfluß dieser Abwässer auf einige charakteristische Vertreter der Primärproduzenten untersucht. Sind es doch gerade die Primärproduzenten, die als Anfangsglied der Nahrungskette maßgebenden Einfluß auf den Stoffhaushalt des Meeres haben.

\section{MATERIAL UND METHODE}

Für die Versuche wurden zwei Arten von Dinoflagellaten aus dem Helgoländer Plankton isoliert und in Kultur genommen: Prorocentrum micans und Ceratium furca. Die Kulturen wurden artrein (Unialgal-Kultur) gezüchtet, aber nicht bakterienfrei (axenisch) gehalten.

Beide Arten wurden zunächst in Schreiberlösung (Seewasser $+10 \% \mathrm{NaNO}_{3}+$ $2 \% \mathrm{Na}_{2} \mathrm{HPO}_{4}$ ) kultiviert und bei $6000 \mathrm{Lux}$ (Tageslicht-Leuchtröhren) und $18^{\circ} \mathrm{C}$ in 5-Liter-Flaschen in Massenkultur genommen. Die Vermehrungsrate wurde durch Auszählung definierter Proben mit dem Plankton-Mikroskop nach Utermöhl bestimmt

Zur Ermittlung optimaler Kulturbedingungen wurde Prorocentrum micans außer in Schreiberlösung auch in Erdschreiberlösung (Schreiberlösung $+20 \%$ Erdextrakt) und in einer halbsynthetischen Nährlösung (Seewasser $=1020 \mathrm{~g} ; \mathrm{NaNO}_{3}=42,5 \mathrm{mg}$; $\mathrm{Na}_{2} \mathrm{HPO}_{4} \cdot 12 \mathrm{H}_{2} \mathrm{O}=10,75 \mathrm{mg} ; \mathrm{FeSO}_{4} \cdot 7 \mathrm{H}_{2} \mathrm{O}=0,278 \mathrm{mg} ; \mathrm{MnCl}_{2} \cdot 4 \mathrm{H}_{2} \mathrm{O}=$ $0,0198 \mathrm{mg} ; \mathrm{Na} 2 \mathrm{EDTA} \cdot 2 \mathrm{H}_{2} \mathrm{O}=3,72 \mathrm{mg}$; Vitamin $\mathrm{B}_{12}=0,0007 \mathrm{mg}$ (abgeändert nach v. Sтоsch \& Drebes 1964) gezüchtet. Diese Versuche wurden bei $10^{\circ} \mathrm{C}, 14^{\circ} \mathrm{C}$ und $18^{\circ} \mathrm{C}$ in Flaschen mit je $200 \mathrm{~cm}^{3}$ Nährlösung durchgeführt.

Zusätzlich zu den Kulturversuchen in Flaschen mit konstantem Volumen wurde die Vermehrungsrate der Algen auch in Kulturen mit ansteigendem Volumen bestimmt. Die Menge der täglich zugesetzten Nährlösung wurde so bemessen, daß der tägliche Zuwachs an Zellen durch Verdünnung wieder ausgeglichen wurde und somit die Zelldichte der Kultur konstant blieb. Dieses Verfahren entspricht dem Chemostat-Prinzip und wird im folgenden als "Chemostat mit steigendem Volumen " bezeichnet. Die Kulturen wurden auf einer Zelldichte von $1000 \mathrm{Zellen} / \mathrm{cm}^{3}$ gehalten. Die Versuche wurden von einem Anfangsvolumen von $250 \mathrm{~cm}^{3}$ ausgehend bei zum Erreichen der 6-LiterGrenze durchgeführt (Schreiberlösung, $18^{\circ} \mathrm{C}, 6000 \mathrm{Lux}$, Tageslicht-Leuchtröhren).

Für die Versuche mit industriellem Abwasser wurde eine von der Titangesellschaft m.b.H. zur Verfügung gestellte Abwasserprobe verwendet. Nach Angabe der Firma setzt sich das Abwasser aus Dünnsäure und Grünsalz im Mengenverhältnis von 10 Liter Dünnsäure auf $3,3 \mathrm{~kg}$ Grünsalz zusammen und soll je nach Jahreszeit mit Temperaturen zwischen $20^{\circ}$ und $40^{\circ} \mathrm{C}$ in See eingeleitet werden. Die Zusammensetzung der Dünnsäure ist aus Tabelle 1 ersichtlich. Das Abwasser soll ab Anfang 1969 mit einem Spezialschiff in Mengen von etwa 850 bis 880 Tonnen Dünnsäure und etwa 350 Tonnen Grünsalz pro Tag 12 Seemeilen nordwestlich Helgolands verklappt werden. 
Im unverdünnten Abwasser ist bei Zimmertemperatur ein Teil des $\mathrm{FeSO}_{4}$ auskristallisiert. $\mathrm{Um}$ alles $\mathrm{FeSO}_{4}$ in Lösung zu bringen, wurde das Abwasser auf $70^{\circ} \mathrm{C}$ erhitzt und eine Stammlösung von 1 Teil Abwasser auf 10 Teile Seewasser hergestellt. Zum Ansetzen der Verdünnungsreihen wurde die Stammlösung im Bereich von 1:2500 bis 1:100000 Teilen Abwasser zu Seewasser gemischt. Das Seewasser (32\% \pm $1,5 \% \mathrm{~S}$ ) wurde der Helgoländer Reede entnommen und zur Anreicherung der Nährstoffe mit $\mathrm{NaNO}_{3}(10 \%)$ und $\mathrm{Na}_{2} \mathrm{HPO}_{4}(2 \%)$ versetzt.

Tabelle 1

Zusammensetzung der Abwässer der Titangesellschaft m.b.H., Leverkusen

\begin{tabular}{|c|c|c|}
\hline Dünnsäure & $\begin{array}{l}\mathrm{H}_{2} \mathrm{SO}_{4} \\
\mathrm{FeSO}_{4} \\
\mathrm{TiOSO}_{4} \\
\mathrm{MnSO}_{4} \\
\mathrm{MgSO}_{4} \\
\mathrm{Cr}_{2}\left(\mathrm{SO}_{4}\right)_{3} \\
\mathrm{VoSO}_{4} \\
\mathrm{Al}_{2}\left(\mathrm{SO}_{4}\right)_{3} \\
\mathrm{Na}_{2} \mathrm{O} \\
\mathrm{CaSO}_{4} \\
\mathrm{H}_{2} \mathrm{O} \text { (Rest) }\end{array}$ & $\begin{array}{rr}14,4 & \% \\
7,1 & \% \\
1,6 & \% \\
0,08 & \% \\
1,59 & \% \\
0,02 & \% \\
0,065 & \% \\
0,3 & \% \\
0,04 & \% \\
0,1 & \%\end{array}$ \\
\hline Grünsalz & $\begin{array}{l}\mathrm{FeSO}_{4} \\
\mathrm{H}_{2} \mathrm{SO}_{4} \\
\mathrm{TiOSO}_{4} \\
\mathrm{MgSO}_{4} \\
\mathrm{MnSO}_{4} \\
\mathrm{H}_{2} \mathrm{O}\end{array}$ & $\begin{array}{r}50 \% \\
0,25 \% \\
0,4 \% \\
2,5 \% \\
0,09 \% \\
46,76 \%\end{array}$ \\
\hline
\end{tabular}

Bei Einleitung von frischem Titanwerk-Abwasser in die See finden lebhafte chemisch-physikalische Umsetzungen mit dem Meerwasser statt. Das schwefelsäurehaltige Abwasser, das etwa einer 4-normalen $\mathrm{H}_{2} \mathrm{SO}_{4}$-Lösung entspricht, läßt den $\mathrm{pH}$-Wert des Seewassers, der etwa bei $\mathrm{pH} 8$ liegt, je nach Konzentration rasch absinken. Die Schwefelsäure zerstört dabei das Kalk-Kohlensäure-Gleichgewicht des Seewassers und führt zu einem schnellen Anstieg des $\mathrm{CO}_{2}$-Partialdrucks. Erst die Turbulenz des Seewassers läßt das $\mathrm{CO}_{2}$ in die freie Luft entweichen, so daß sich ein neues Gleichgewicht einstellt. Entsprechend steigt der $\mathrm{pH}$-Wert des Wassers wieder an.

Die Eisenionen fallen im schwach alkalischen Seewasser als flockiger Eisenhydroxid-Niederschlag aus. Mit dieser Reaktion ist eine Sauerstoffzehrung verbunden. Die Reaktion läßt sich durch die Formel $2 \mathrm{Fe}^{++}+4 \mathrm{OH}^{\prime}+\mathrm{H}_{2} \mathrm{O}+1 / 2 \mathrm{O}_{2} \rightarrow 2 \mathrm{Fe}(\mathrm{OH})_{3}$ ausdrücken.

Die Reaktionen des Abwassers mit dem Seewasser bestimmten die Methodik der Versuchsanordnung. Parallel zur Messung der Vermehrungsrate der Organismen durch Zählung wurden pH-Messungen in den Versuchsgefäßen durchgeführt. Turbulenz und Durchlüftung des Seewassers wurde durch grobblasige Belüftung nachgeahmt. Die Temperatur in den Versuchsgefäßen betrug $18^{\circ} \mathrm{C}$, die Beleuchtung $2 \times 3000$ Lux zweier Tageslicht-Leuchtröhren, die seitlich der Versuchsflaschen angeordnet waren. 
Um den unterschiedlichen Verhältnissen, denen die Organismen bei der Abwassereinleitung auf offener See ausgesetzt sind, möglichst nahezukommen, wurden die Versuche nach drei verschiedenen Methoden durchgeführt: (1) Die Organismen geraten in eine in See treibende Titan-Abwasserwolke hinein. Für die Versuche wurden die Testorganismen in ein um 24 Stunden gealtertes Abwasser-Seewasser-Gemisch eingesetzt. Die Reaktionen des Abwassers mit dem Seewasser waren in dieser Zeit bereits abgelaufen: das Kalk-Kohlensäure-Gleichgewicht des Gemisches hatte sich wieder eingestellt. Der Eisenhydroxidniederschlag war ausgefallen. Die Testorganismen wurden in verschiedenen Abwasserkonzentrationen in 200- $\mathrm{cm}^{3}$-Flaschen über mehrere Wochen hinweg weiterkultiviert. Diese Versuche wurden mit beiden Testarten durchgeführt. (2) Die Testorganismen geraten direkt in frischeingeleitetes Abwasser hinein und nehmen einmal an der Reaktion des Abwassers mit dem Seewasser teil. Im Versuch befanden sich die Organismen daher schon im Versuchsgefäß, während die entsprechende $\mathrm{Abwassermenge} \mathrm{zugesetzt} \mathrm{wurde,} \mathrm{und} \mathrm{durchliefen} \mathrm{sowohl} \mathrm{die} \mathrm{anfänglich} \mathrm{starke} \mathrm{pH-}$ Erniedrigung als auch die Fällung des Eisenhydroxidniederschlages. Die Testorganismen wurden gleichfalls in $200-\mathrm{cm}^{3}$-Flaschen über mehrere Wochen hinweg weiterkultiviert. Diese Versuche wurden nur mit Prorocentrum micans durchgeführt. (3) Die Testorganismen werden täglich mit frischem Abwasser konfrontiert. Für diese Versuche eignete sich am besten das Chemostat-Verfahren, bei dem die Kultur durch tägliche Verdünnung mit Kulturlösung auf einer konstanten Zelldichte gehalten wurde. Die Menge der täglich zur Verdünnung zugesetzen Lösung wurde aus der Zuwachsrate im Kontrollversuch berechnet. Die gleiche Menge an Lösung, jedoch mit frischem Abwasser der Titanwerke versetzt, wurde den entsprechenden Abwasserversuchsgefäßen täglich zugesetzt.

Die Versuche nach dem Chemostat-Prinzip wurden nach zwei Methoden durchgeführt: (a) Chemostat mit steigendem Volumen. Entsprechend der auf p. 23 bereits ausgeführten Methode wurde dem Kontrollversuch täglich soviel neue Nährlösung zugesetzt, daß die Zelldichte auf 1000 Zellen $/ \mathrm{cm}^{3}$ konstant gehalten wurde. Den Abwasserversuchsgefäßen wurde die gleiche Menge an frischem Abwasser-Seewasser-Gemisch zugesetzt. Von $250 \mathrm{~cm}^{3}$ ausgehend, wurden die Versuche nach Erreichen der 5-LiterGrenze beendet (15 Tage). Die gesamte Population, die im Versuchsgefäß enthalten ist, bleibt über die Versuchszeit hinweg erhalten. (b) Chemostat mit konstantem Volumen. Auch hier wurde die Zelldichte im Kontrollversuch auf $1000 \mathrm{Zellen} / \mathrm{cm}^{3}$ konstant gehalten. Das Versuchsvolumen blieb dagegen für die ganze Dauer des Versuchs auf 1 Liter in jeder Flasche beschränkt. Hierfür wurde der Kontrollkultur zunächst die Menge an Lösung entnommen, die den täglichen Zuwachs an Zellen enthielt und anschließend das Versuchsgefäß mit frischer Nährlösung wieder auf einen Liter aufgefüllt. In den Abwasserversuchsgefäßen wurde der Wasseraustausch in gleicher Höhe vollzogen, mit dem Unterschied, daß die zum Auffüllen zugesetzte Lösung die entsprechende Menge an frischem Titanwerke-Abwasser enthielt. Das Verfahren erlaubte eine beliebig lange Fortsetzung der Versuche und wurde über einen Zeitraum von 25 Tagen durchgeführt. Im Gegensatz zum Versuch (a) wird der Population des Kontrollversuchs täglich die dem Zuwachs entsprechende Anzahl an Zellen entzogen. In entsprechendem Maße werden auch die Populationen in den Abwasserversuchsgefäßen reduziert. 


\section{ERGEBNISSE}

\section{Prorocentrum micans}

\section{Versuche zur Ermittlung optimaler Kulturbedingungen}

Abbildung 1 zeigt die Ergebnisse von Zuchtexperimenten in Kulturen mit begrenztem Volumen. Bei $18^{\circ} \mathrm{C}$ wachsen die Kulturen in Schreiberlösung in ca. 30 Tagen von einer Zelle $/ 10 \mathrm{~cm}^{3}$ auf eine Dichte von 30000 Zellen/10 $\mathrm{cm}^{3}$ heran. Bei halblogarithmischer Auftragung verläuft die Wachstumskurve in Gestalt einer Geraden. Ganz ähnlich verhalten sich die Wachstumskurven in Erdschreiberlösung und in halbsynthetischer Lösung: Mit einer Verzögerung von ca. 2 Tagen wachsen die Kulturen bis $z u$ einer Zelldichte von 20000 Zellen heran. Aus dem Anstieg der Geraden läßt sich eine mittlere Teilungsgeschwindigkeit von je einer Zellteilung in 2 Tagen ablesen. Nach ca. 30 Tagen ist die Nährlösung in den Kulturflaschen erschöpft; die Kulturen haben das Maximum ihrer Dichte erreicht und beginnen abzusterben. Im Verlauf weiterer 12 Tage sinkt daher die Zelldichte in allen drei Kulturen auf ca. 1000 Zellen $/ 10 \mathrm{~cm}^{3} \mathrm{ab}$. Bis hierhin entsprechen die Kurven dem charakteristischen Wachstumsschema einzelliger Algen von Fogg (1965). Uberraschenderweise kommen die Absterbeprozesse in der Phase der sinkenden relativen Wachstumsrate jedoch nach etwa 12 Tagen zum Stehen. Auf Grund von Remineralisierungsvorgängen aus den abgestorbenen Zellen werden crneut die ins Minimum geratenen Nährstoffe frei und ermöglichen eine Erholung der Kulturen. Nach insgesamt 65 Tagen hat die Zelldichte in halbsynthetischer Nährlösung die Zahl von 10000 Zellen/10 $\mathrm{cm}^{3}$ wieder erreicht. Die Kulturen in Schreiber- und Erdschreiberlösung folgten mit 8000 und 4500 Zellen $/ 10 \mathrm{~cm}^{3}$.

Unter den extremen Bedingungen eines auf $200 \mathrm{~cm}^{3}$ beschränkten Lebensraums in der Kulturflasche gelingt es Prorocentrum micans, über lange Zeiträume hinweg ein Gleichgewicht zwischen Absterberate und Zuwachsrate herzustellen und ein kleines, in sich geschlossenes Okosystem aufzubauen. Minima und Maxima der Populationsdichte folgen vermutlich wellenförmig hintereinander.

Bei $14^{\circ} \mathrm{C}$ verlaufen die Wachstumskurven in den drei Nährstofflösungen in gleicher Weise annähernd parallel, erreichen aber erst nach ca. 55 Tagen ihre Maxima. Für den Anstieg ergibt sich eine mittlere Teilungsgeschwindigkeit von je einer Zellteilung in 4 Tagen. Die Zelldichte erreicht Werte von 20000 Zellen $/ 10 \mathrm{~cm}^{3}$ in halbsynthetischer Lösung und 12000 bis 14000 Zellen $/ 10 \mathrm{~cm}^{3}$ in Schreiber- und Erdschreiberlösung. Remineralisierungsvorgänge konnten noch nicht erfaßt werden, da der Versuch nach 65 Tagen beendet wurde.

Bei $10^{\circ} \mathrm{C}$ beginnt das Wachstum der Kultur in Schreiberlösung sehr zögernd und erreicht nach 65 Tagen erst eine Zelldichte von 1500 Zellen/10 $\mathrm{cm}^{3}$. Der Anstieg der Kurve entspricht einer mittleren Teilungsgeschwindigkeit von je einer Teilung in 6 Tagen. In den beiden anderen Medien verläuft das Wachstum so unregelmäßig und verzögert, daß auf eine Darstellung der Kurven verzichtet wurde.

Aus den Versuchen läßt sich erkennen, daß die drei verwendeten Nährlösungen etwa gleich gute Wachstumsbedingungen bieten. Der Temperaturfaktor ist deutlich 


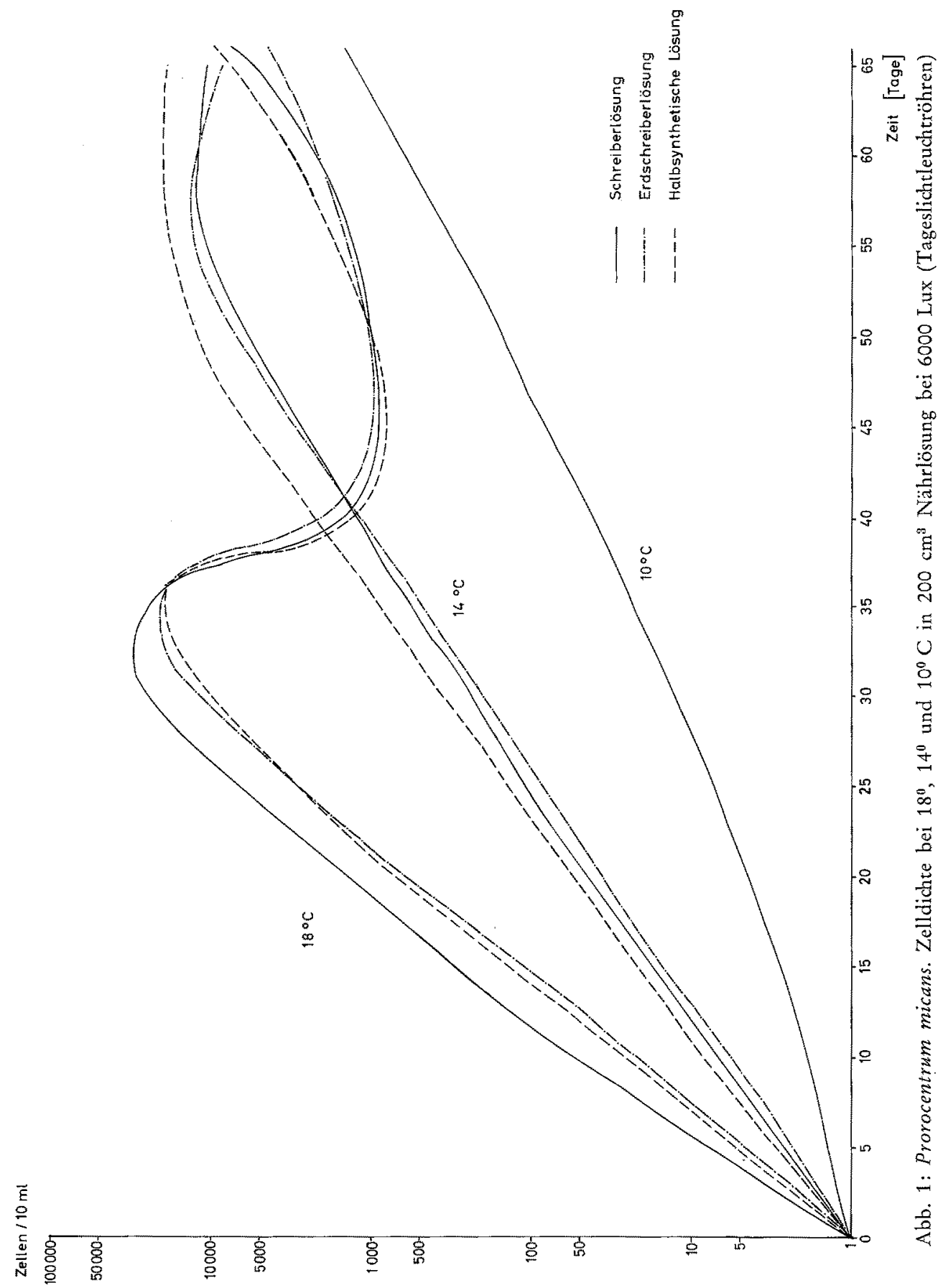


ausgeprägt und zeigt in unseren Versuchen bei $18^{\circ} \mathrm{C}$ die besten Bedingungen. Nach Branrud (1961) liegt das Temperaturoptimum für Prorocentrum micans bei $20^{\circ} \mathrm{C}$.

Die Ergebnisse der Zuchtexperimente im Chemostat-Verfahren mit steigendem Volumen (vgl. p. 23) sind in Abbildung 2 dargestellt. Die rechte Ordinate der Abbildung zeigt das Versuchs-Volumen an, die linke die Gesamtzellzahl im Versuchsgefäß. Die Wachstumskurve der Population verläuft bei halblogarithmischer Darstellung als Gerade. Die Zellzahl steigt von 250000 Zellen in $250 \mathrm{~cm}^{3}$ nach 10 Tagen auf 6000000 Zellen in einem Volumen von 6 Litern an. Die Vermehrung entspricht je einer Zellteilung in ca. 2,2 Tagen. Der tägliche Zuwachsfaktor beträgt 1,39. Das Verfahren ermöglicht es, die Kultur über die ganze Versuchsdauer in der exponentiellen (logarithmischen) Phase zu halten.

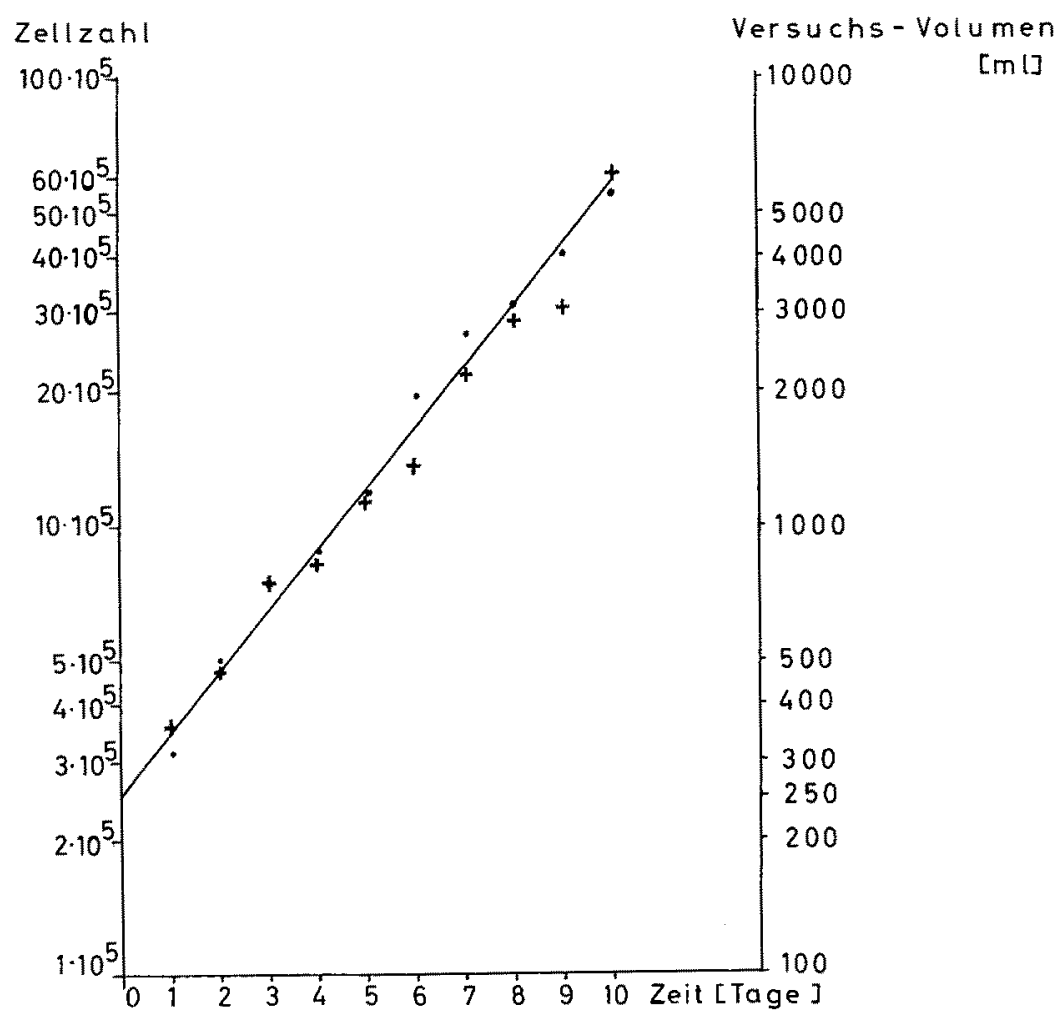

Abb. 2: Prorocentrum micans. Zunahme der Zellzahl im "Chemostat mit steigendem Volumen" bei konstanter Zelldichte von 1000 Zellen $/ \mathrm{cm}^{3}$ (Schreiberlösung, $18^{\circ} \mathrm{C}, 6000$ Lux Tageslichtleuchtröhren)

\section{Zuchtexperimente unter Abwassereinflu $\beta$}

Auf Grund der vorstehend geschilderten Ergebnisse zeigt sich, daß Prorocentrum micans in reiner Schreiberlösung gute Wachstumsbedingungen findet. Für die Ver- 
suche unter Abwassereinfluß wurde daher diese Nährlösung zugrunde gelegt. Erdschreiberlösung und Nährlösungen mit Zusatz von Metallchelatoren (EDTA) eignen sich nicht für die Toxizitätsuntersuchungen, da sie die im Abwasser enthaltenen giftigen Schwermetallionen abpuffern und somit dem Wirkungsmechanismus entziehen.

Die Ergebnisse der Wachstumsversuche in einem um 24 Stunden gealterten Abwasser-Seewasser-Gemisch (vgl. p. 25) sind in Abbildung 3 dargestellt. Die Kulturen enthalten zu Versuchsbeginn 20 Zellen $/ \mathrm{cm}^{3}$. Im Kontrollversuch ohne Abwasserzusatz wachsen die Zellen innerhalb von 14 Tagen auf die Dichte von $1200 \mathrm{Zellen} / \mathrm{cm}^{3}$ heran.

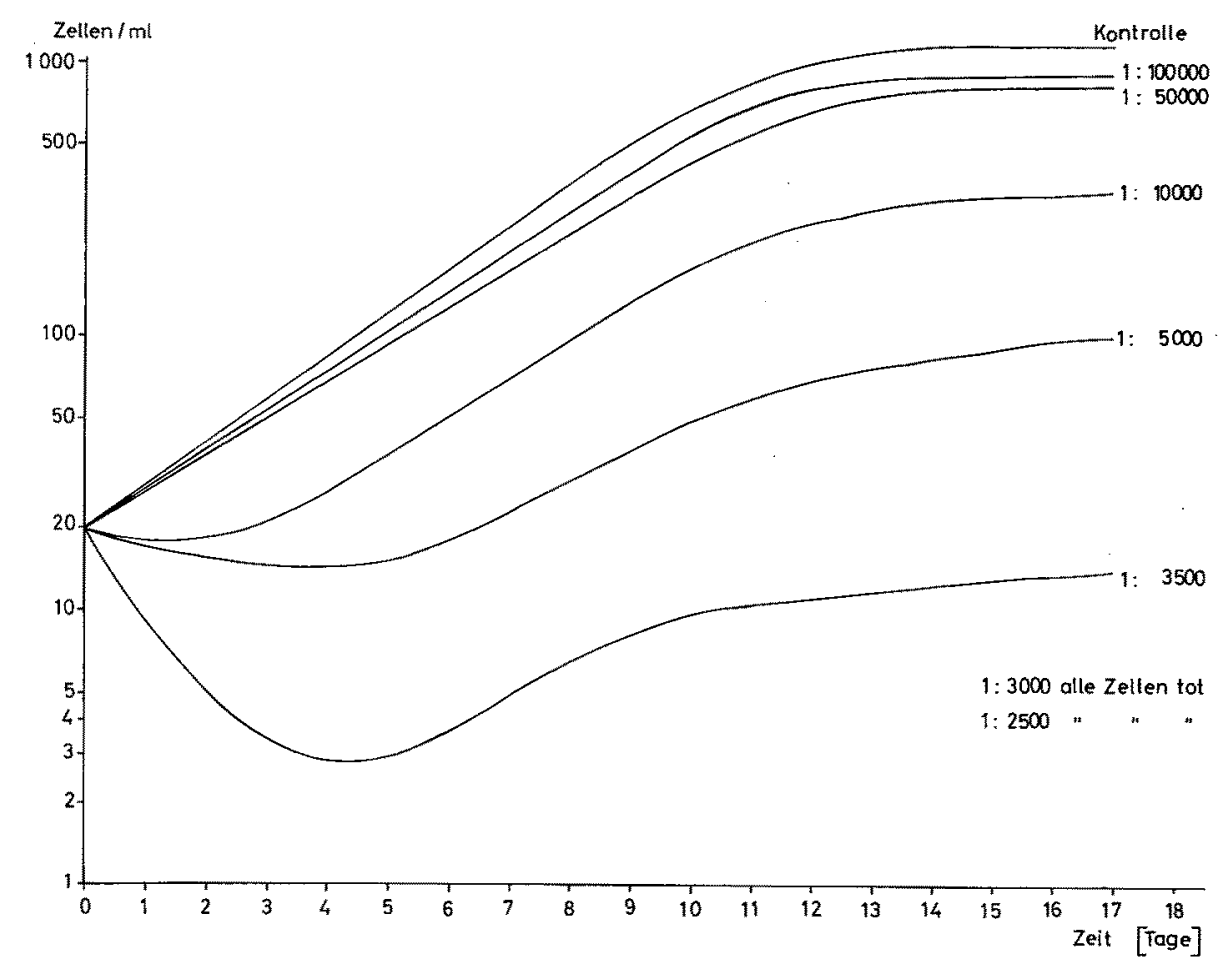

Abb. 3: Prorocentrum micans. Verlauf der Wachstumskurven bei Einwirkung von um 24 Stunden gealterten Abwasser-Seewassergemischen in $200 \mathrm{~cm}^{3}$ Lösung.

In Abwasserkonzentrationen von 1: 100000 und 1:50 000 Teilen Abwasser zu Schreiberlösung zeigen die Kulturen jeweils eine geringfügige Verzögerung der Vermehrungsrate und wachsen bis zur Beendigung der Versuche nach 17 Tagen nur auf Zelldichten von 900 und $800 \mathrm{Zellen} / \mathrm{cm}^{3}$ heran. Von Konzentrationen von 1:10 000 an macht sich bereits in den ersten Tagen eine deutliche Schädigung bemerkbar. Die Kulturen erholen sich jedoch nach einigen Tagen. So verläuft die Wachstumskurve der Population bei 1:10000 vom 4. Tag an den vorherigen Kurven parallel, erreicht aber bei Versuchsende nur eine maximale Zelldichte von ca. $350 \mathrm{Zellen} / \mathrm{cm}^{3}$. Bei der Konzentration 1:5000 wird nach anfänglichem Absinken der Zellzahl auf 15 Zellen/ $\mathrm{cm}^{3}$ bei 


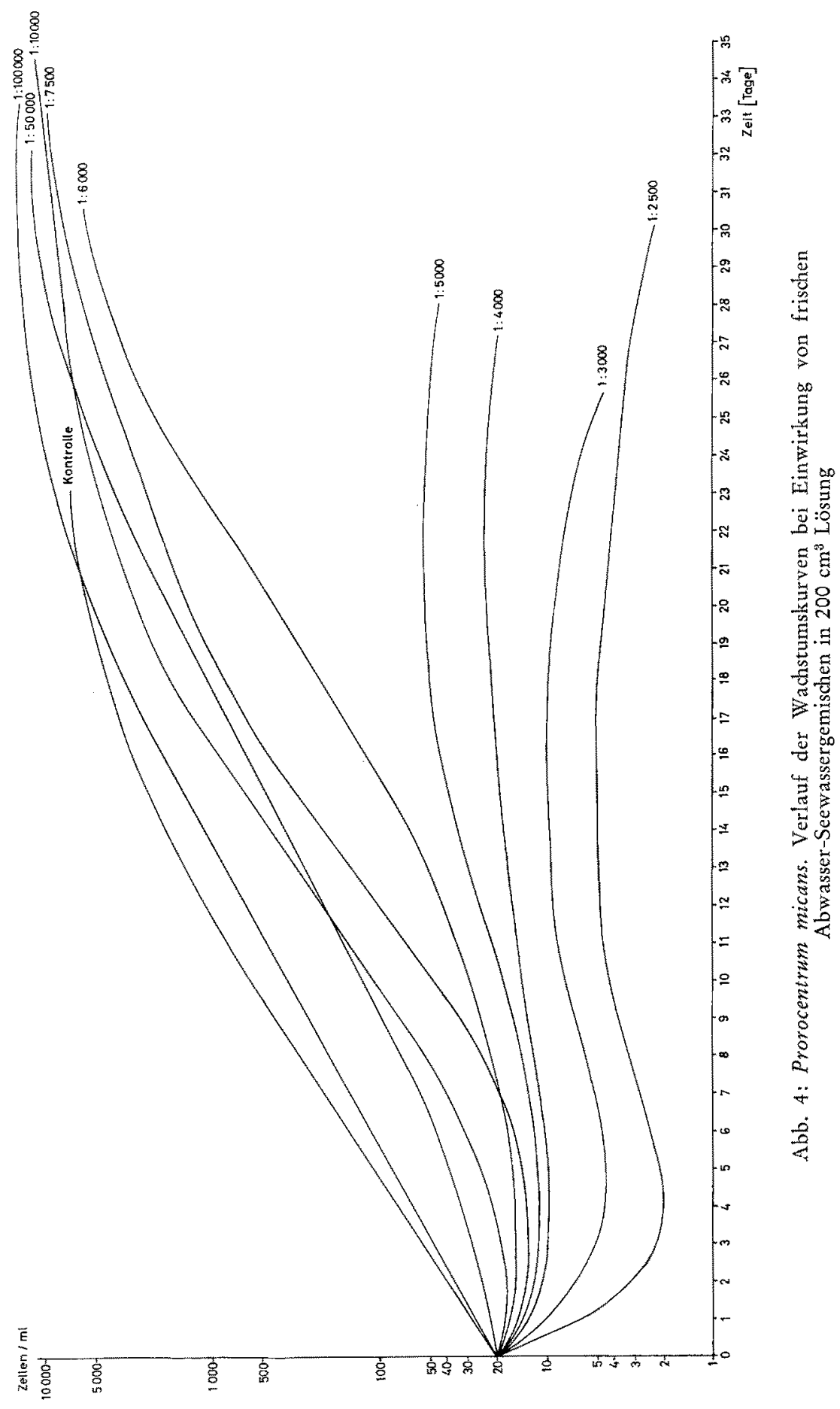




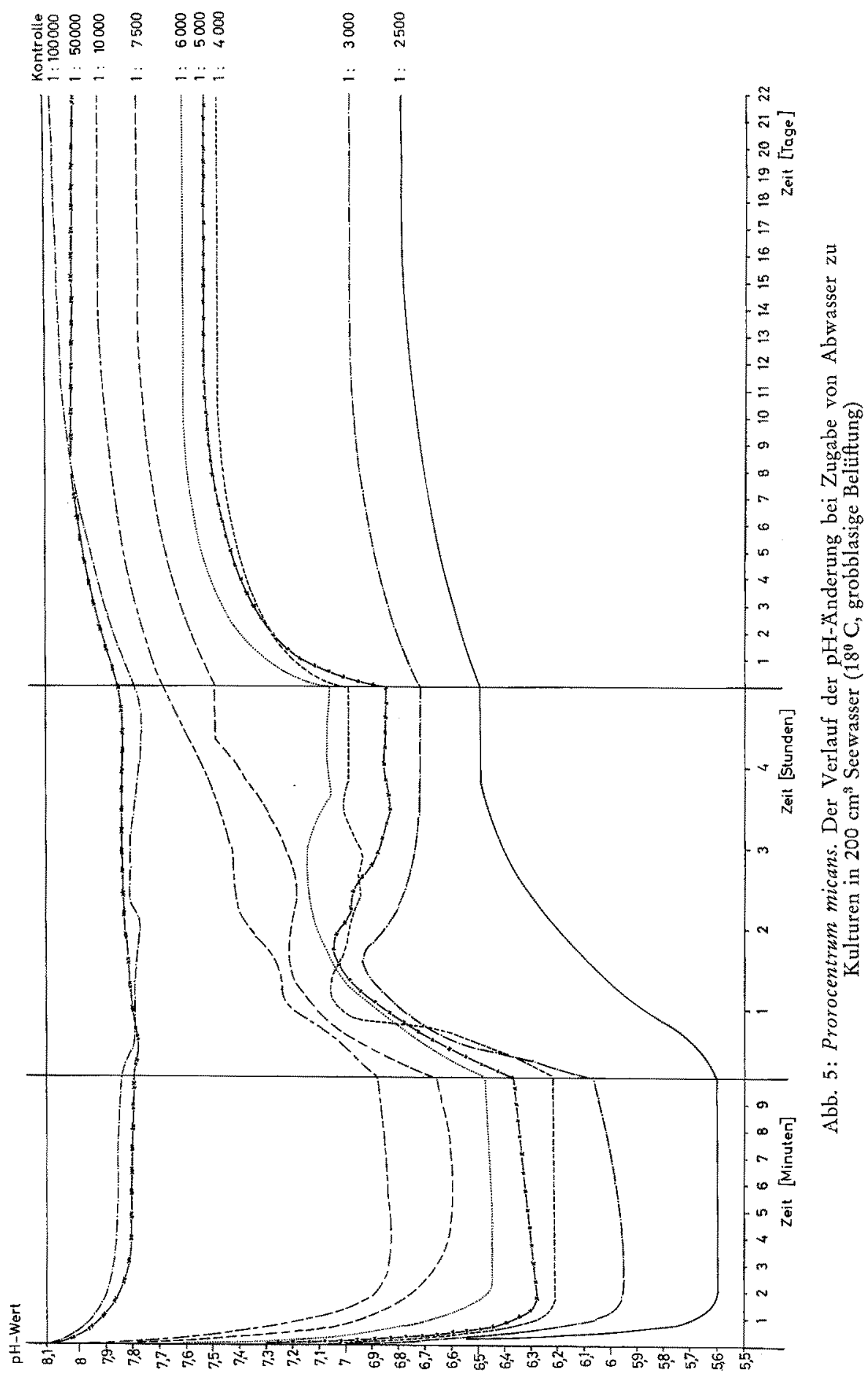


Versuchsende nur noch eine Dichte von $100 \mathrm{Zellen} / \mathrm{cm}^{3}$ erreicht. Die Konzentration von $1: 3500$ führt fast zum Absterben der Kultur: ausgehend von $20 \mathrm{Zellen} / \mathrm{cm}^{3}$ sinkt die Zellzahl nach 4 Tagen auf 3 Zellen $/ \mathrm{cm}^{3} \mathrm{ab}$. Nach leichter Erholung in den nächsten 5 Tagen wird bei Versuchsende nur eine Zellzahl von ca. $15 \mathrm{Zellen} / \mathrm{cm}^{3}$ erreicht. In Abwasserkonzentrationen von $1: 3000$ und $1: 2500$ sterben die Kulturen in diesem Versuch $a b$.

Die Ergebnisse der Versuche mit frisch eingeleitetem Abwasser zeigen Abbildungen 4 und 5. Den Testorganismen wird bei Versuchsbeginn einmal frisches Abwasser zugesetzt. Wie im Versuch mit gealtertem Abwasser-Seewasser-Gemisch enthalten die Kulturen zunächst $20 \mathrm{Zellen} / \mathrm{cm}^{3}$ (Abb. 4). In der Kontrolle wird nach 22 Tagen eine Zelldichte von 7000 Zellen $/ \mathrm{cm}^{3}$ erreicht. Bei der Abwasserkonzentration von 1:100000 folgt die Kurve der Vermehrungsrate der Kontrollkurve mit leichter Verzögerung, übergipfelt sie aber vom 21. Tage an und erreicht nach 33 Tagen bei Versuchsende den Wert von $15000 \mathrm{Zellen} / \mathrm{cm}^{3}$. Eine immer deutlicher ausgeprägte Anfangsschädigung zeigen die Konzentrationen von $1: 50000,1: 10000,1: 7500$ und 1:6000. Bei den 3 letzteren ist die Absterberate in den ersten Tagen sogar größer als die Zuwachsrate. In allen Verdünnungsstufen tritt jedoch eine Erholung ein, die bei Versuchsende nach ca. 32 Tagen zu Zelldichten von $12000(1: 50000$ und $1: 10000), 10000(1: 7500)$ und $6000(1: 6000)$ Zellen $/ \mathrm{cm}^{3}$ führt. Bei Konzentrationen von $1: 5000$ findet nach anfänglichem Rückgang der Population nur noch ein sehr geringer und bei 1:4000 kein Zuwachs an Zellen mehr statt. Nach 27 Tagen enthalten die Kulturen nur 50 bzw. 20 Zellen $/ \mathrm{cm}^{3}$ und zeigen eine rückläufige Tendenz der Vermehrungsrate. Bei Konzentrationen von $1: 3000$ und $1: 2500$ sterben die Kulturen nach 4 bis 5 Tagen bis auf wenige Individuen $a b$. Die Überlebenden teilen sich zögernd weiter, erreichen aber nur Zelldichten von $10 \mathrm{bzw} .5$ Zellen/ $\mathrm{cm}^{3}$ nach ca. 16 Tagen. Im weiteren Verlauf des Versuchs gehen die Kulturen zahlenmäßig wieder leicht zurück. Trotzdem sind in der Verdünnungsstufe von $1: 2500$ nach 30 Tagen noch einige Individuen lebend nachzuweisen.

Abbildung 5 zeigt die zu den verschiedenen Abwasserkonzentrationen gehörigen $\mathrm{pH}$-Werte. Um den Verlauf der $\mathrm{pH}$-Anderung in Abhängigkeit von der Zeit in allen Einzelheiten über die ganze Versuchsdauer hinweg darzustellen, mußte die Zeitskala in drei verschiedene Maßstäbe unterteilt werden. Abschnitt 1 ist in Minuten unterteilt und umfaßt die Zeit vom Augenblick der Abwasserzugabe bis zum Ablauf der ersten 10 Minuten. Abschnitt 2 zeigt den Verlauf der pH-Anderung in den anschließenden nächsten 5 Stunden und Abschnitt 3 in den folgenden 22 Tagen an.

Die den.Versuchen zugrunde gelegte Schreiberlösung hat einen $\mathrm{pH}$ von 8,12 . Im Kontrollversuch bleibt dieser Wert relativ konstant über die ganze Versuchsdauer hinweg erhalten. In den Versuchen mit Abwasserzugabe fällt der $\mathrm{pH}$-Wert in den ersten Minuten nach der Einleitung sofort ab. Im Mischungsverhältnis von 1:100000 und 1: 50000 sinkt er von 8,12 nach 4 Minuten auf 7,85 bzw. 7,80 ab und halt diesen Wert mit leichten Schwankungen auch über die nächsten 5 Stunden ein. Im Verlauf der nächsten Tage steigt er wieder auf 8,13 bzw. 8,05 an und bleibt auf diesem Wert stehen. In der Konzentration von $1: 10000$ sinkt der $\mathrm{pH}$-Wert nach 4 Minuten auf 6,83 ab. Nach einer Stunde steigt er wieder bis auf 7,20 und nach 5 Stunden auf 7,70 an. Bei Versuchsende nach 22 Tagen hat er sich auf 7,95 eingestellt. In den höheren Konzentrationsstufen wird der pH-Abfall in den ersten Minuten immer ausgeprägter und erreicht bei 
$1: 2500$ den Wert von 5,6 nach 2 Minuten. In der folgenden Phase des pH-Anstiegs macht sich in den Konzentrationen von $1: 7500$ bis $1: 3000$ jedoch eine deutlich ablesbare Tendenz bemerkbar: Der Anstieg kommt nach 1 bis 2 Stunden zum Stillstand und sinkt in den anschließenden 3 bis 4 Stunden wieder etwas ab. Besonders deutlich ist das Verhalten in der Konzentration von 1:3000 ausgeprägt, in der der pH-Wert nach 1 Stunde und 40 Minuten ein Maximum von 6,94 erreicht und in den folgenden 2 Stunden wieder auf 6,73 zurückgeht. Die Maxima fallen mit dem Ausfall des Eisenhydroxidniederschlages zusammen. Der unruhige Kurvenverlauf kennzeichnet die Phase der als Zeitreaktionen ablaufenden chemisch-physikalischen Umsetzungen des Abwassers mit dem Seewasser. 4 bis 5 Stunden nach Zugabe des Abwassers hat sich wieder ein neues Gleichgewicht eingestellt. Die Konzentration von $1: 2500$ folgt nicht diesem Kurvenverlauf, sondern steigt im Verlauf von 4 Stunden von $\mathrm{pH} 5,6$ kontinuierlich auf $\mathrm{pH} 6,5$ an. Der Verlauf der pH-Änderung in den folgenden 22 Tagen zeigt zu Beginn eine leicht steigende Tendenz. Vom 10. Tage an bleibt der pH-Wert jedoch bis zum Versuchsende etwa auf dem gleichen Wert stehen und liegt innerhalb der Werte $7,8(1: 7500)$ und $6,8(1: 2500)$. Die Extremwerte sind in Tabelle 2 zusammengefaßt.

Tabelle 2

pH-Wert bei Zusatz von Abwasser zu $200 \mathrm{~cm}^{3}$ Schreiberlösung

\begin{tabular}{|c|c|c|c|}
\hline $\begin{array}{l}\text { Mischungs- } \\
\text { verhältnis }\end{array}$ & pH-Ausgangswert & $\begin{array}{c}\mathrm{pH}-\text { Minimum } \\
(2-5 \text { min })\end{array}$ & $\begin{array}{l}\text { pH-Endwert } \\
\text { (22 Tage) }\end{array}$ \\
\hline Kontrolle & 8,12 & 8,12 & 8,16 \\
\hline $1: 100000$ & 8,12 & 7,78 & 8,13 \\
\hline $1: 50000$ & 8,12 & 7,78 & 8,04 \\
\hline $1: 10000$ & 8,12 & 6,84 & 7,95 \\
\hline $1: 7500$ & 8,12 & 6,60 & 7,80 \\
\hline $1: 6000$ & 8,12 & 6,45 & 7,63 \\
\hline $1: \quad 5000$ & 8,12 & 6,28 & 7,55 \\
\hline $1: 4000$ & 8,12 & 6,21 & 7,50 \\
\hline 1: 3000 & 8,12 & 5,95 & 7,00 \\
\hline $1: \quad 2500$ & 8,12 & 5,60 & 6,80 \\
\hline
\end{tabular}

Für die Methodik der Versuche im "Chemostat mit steigendem Volumen" sei auf p. 23 und p. 25 verwiesen. Abbildung 6 zeigt das Ergebnis der täglichen Zellzählung in allen 10 Flaschen. Die Kontrolle wird bei einer Dichte von $1000 \mathrm{Zellen} / \mathrm{cm}^{3}$ konstant gehalten. Die Kurve der Vermehrungsrate hat daher die Gestalt einer horizontalen Geraden. In den Verdünnungsstufen des Abwassers im Verhältnis von 1:100000, $1: 50000,1: 32000$ und 1:25000 reagieren die Organismen sofort durch relativ einheitliches Absinken der Zellzahl innerhalb der nächsten 15 Tage. Die Zelldichten bei Versuchsende liegen zwischen 700 und $600 \mathrm{Zellen} / \mathrm{cm}^{3}$. Auch die Konzentrationen $1: 10000$ und $1: 7500$ schließen sich der vorherigen Gruppe noch an, obwohl hier die Zellzahl bereits in den ersten Tagen merklich abnimmt. So sinkt die Zellzahl bei 1: 7500 nach 4 Tagen auf $800 \mathrm{Zellen} / \mathrm{cm}^{3}$, nach 10 Tagen auf $700 \mathrm{Zellen} / \mathrm{cm}^{3}$ und nach 15 Tagen bei Versuchsende auf $550 \mathrm{Zellen} / \mathrm{cm}^{3} \mathrm{ab}$. Scharf abgesetzt gegen die vorige Gruppe verlaufen die Kurven in den Verdünnungsstufen $1: 5000$ und $1: 4000$. Gleich 
in den ersten Tagen sinkt die Zellzahl beträchtlich ab. Nach einem Tag sind noch 700 Zellen $/ \mathrm{cm}^{3}$, nach 3 Tagen nur noch ca. $500 \mathrm{Zellen} / \mathrm{cm}^{3}$ vorhanden. Der rasche Rückgang verlangsamt sich jedoch im Lauf der nächsten Zeitspanne und führt bei Versuchsende zu Zelldichten von ca. $250 \mathrm{Zellen} / \mathrm{cm}^{3}$. In der Konzentration $1: 3000$ sinkt die Zellzahl bis auf $90 \mathrm{Zellen} / \mathrm{cm}^{3}$ nach 15 Tagen ab.

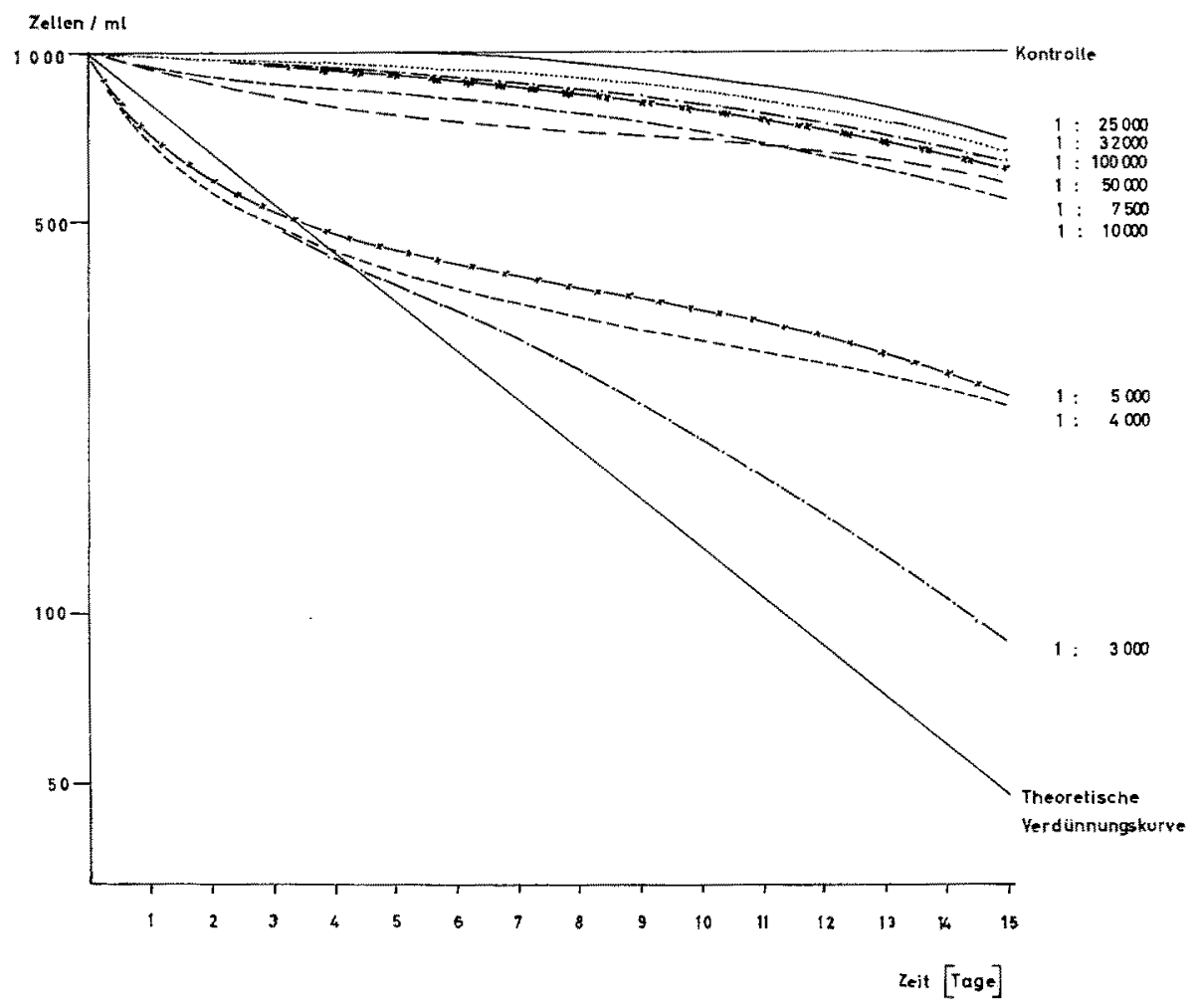

Abb. 6: Prorocentrum micans. Verlauf der Wachstumskurven im "Chemostat mit steigendem Volumen" bei täglichem Zusatz von frischem Abwasser-Seewassergemisch

In Abbildung 6 ist zusätzlich die theoretische Verdünnungskurve eingezeichnet. Sie zeigt die von den Vermehrungsvorgängen unabhängige Abnahme der Zellzahl, die sich aus der täglichen Verdünnung mit zellfreier Versuchslösung ergibt. $1000 \mathrm{Zellen} / \mathrm{cm}^{3}$ werden in 15 Tagen auf $47 \mathrm{Zellen} / \mathrm{cm}^{3}$ verdünnt. Alle oberhalb der Geraden gelegenen Werte zeigen eine Vermehrung, alle unterhalb gelegenen Werte ein Absterben der Kultur an. Parallel zur Geraden verlaufende Kurven bedeuten ein Stagnieren der Kultur auf einer konstanten Zelldichte.

In Abbildung 7 sind die zugehörigen $\mathrm{pH}$-Werte aufgetragen. Im Kontrollversuch bleibt der $\mathrm{pH}$-Wert über die ganze Versuchsdauer hinweg konstant bei etwa 8,01 . In den Abwasserversuchsgefäßen sinkt er am ersten Tag relativ am stärksten ab, da die zugesetzte Abwassermenge auf das gesamte Volumen des ersten Tages $\left(250 \mathrm{~cm}^{3}\right)$ be- 
Züchtungsexperimente an Flagellaten im Abwassertest

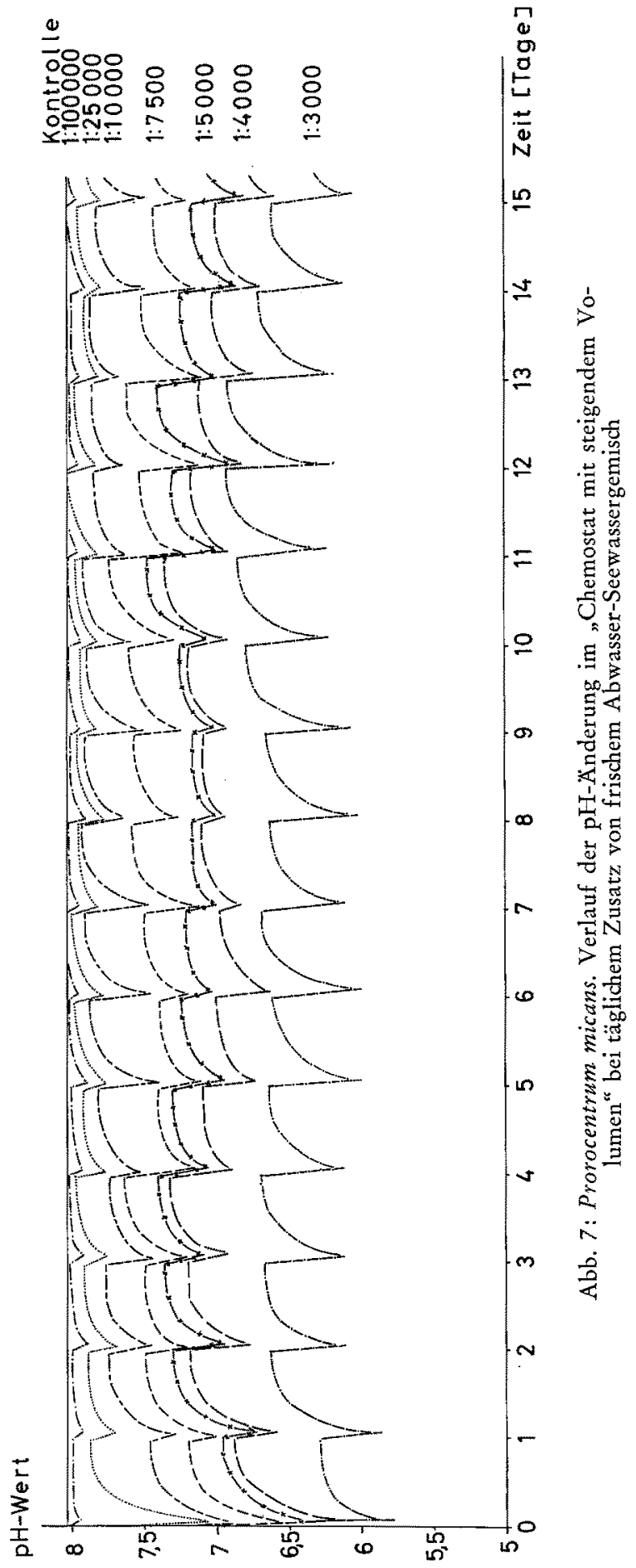


rechnet wurde. An den folgenden Tagen ist der $\mathrm{pH}-$ Sprung geringer, da die zugesetzte Menge an frischem Abwassergemisch jeweils nur etwa einem Fünfel der vorhandenen Menge an bereits gealtertem Abwassergemisch der Vortage entspricht.

In den Konzentrationen von $1: 100000$ bis $1: 25000$ weicht der $\mathrm{pH}$-Wert infolge der täglichen Abwasserbelastung nur um 0,1 bis 0,2 vom täglichen Grundwert ab. Der

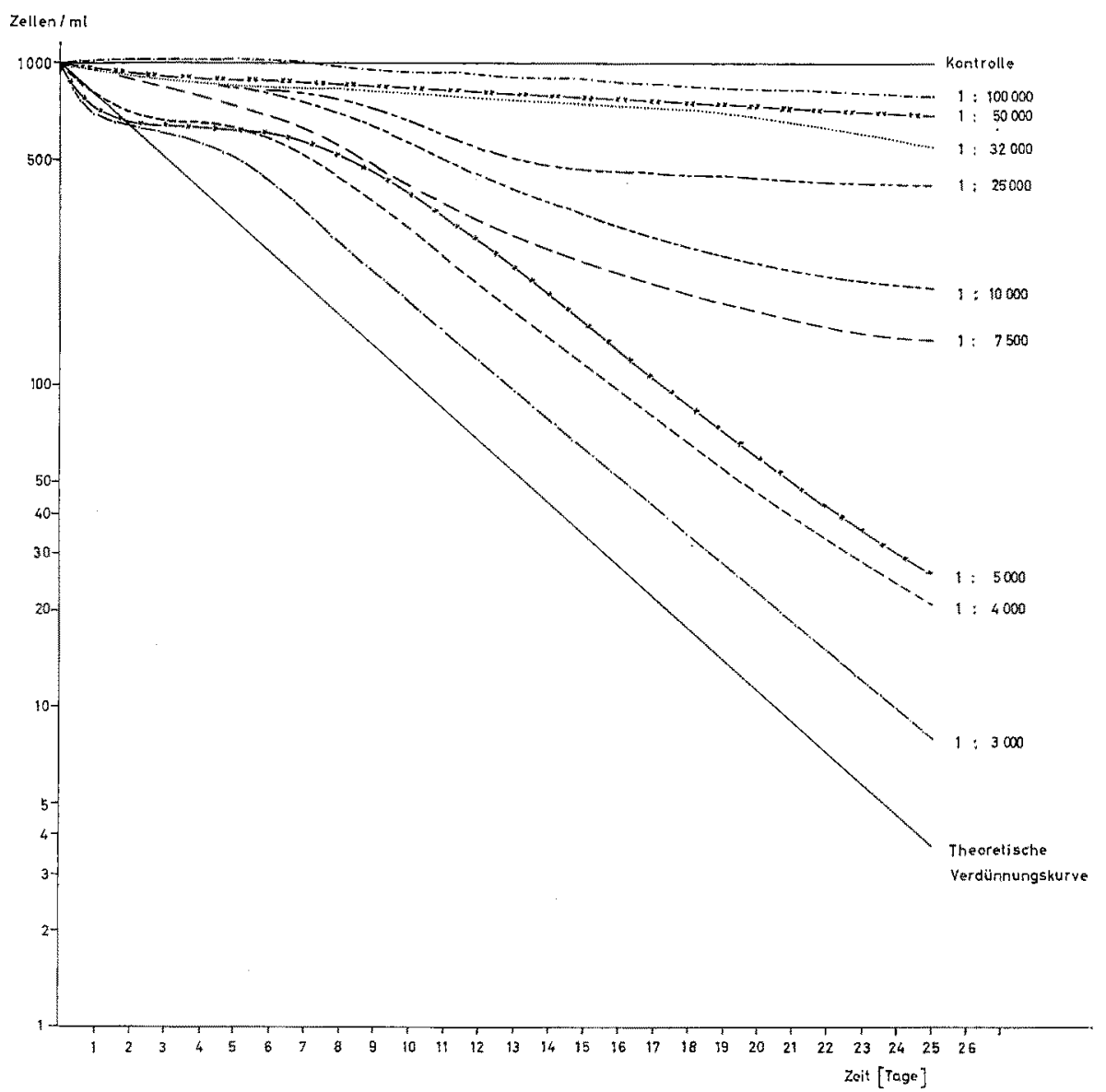

Abb. 8: Prorocentrum micans. Verlauf der Wachstumskurven im "Chemostat mit konstantem Volumen" bei täglichem Zusatz von frischem Abwasser-Seewassergemisch

Grundwert liegt $\mathrm{zwischen} \mathrm{pH} 7,9$ und 8,0. In der Konzentration 1:10000 sinkt der $\mathrm{pH}$-Wert am ersten Tag auf 6,9 ab, stellt sich in den folgenden Tagen aber immer wieder auf einen Grundwert von 7,7 bis 7,9 ein. Die täglichen Abwasserzugaben lassen ihn dabei täglich auf ca. 7,5 absinken. In der Konzentration 1:7500 liegt die Anfangsspitze bei 6,5. Der pH-Wert pendelt in den folgenden Tagen zwischen 7,6 und 7,1. Bei Konzentrationen von $1: 5000$ und $1: 4000$ liegen die täglichen Grundwerte etwa bei $\mathrm{pH} 7,4$ bzw. 7,3; die Spitzenwerte liegen bei pH 7,0 bzw. 6,8. Der tägliche pH-Sprung 
beträgt im Mittel 0,4. In der Konzentration 1:3000 wird am ersten Tage der pH 5,8 erreicht. Die täglich folgenden Spitzenwerte liegen zwischen $\mathrm{pH} 6,0$ und 6,2, gehen jedoch auf Grundwerte von 6,6 bis 6,9 zurück. Die mittlere pH-Schwankung liegt bei 0,6 .

Die Methodik der Versuche im "Chemostat mit konstantem Volumen" wurde auf p. 25 erläutert. In den auf 1 Liter konstant gehaltenen Versuchsflaschen wurden täglich

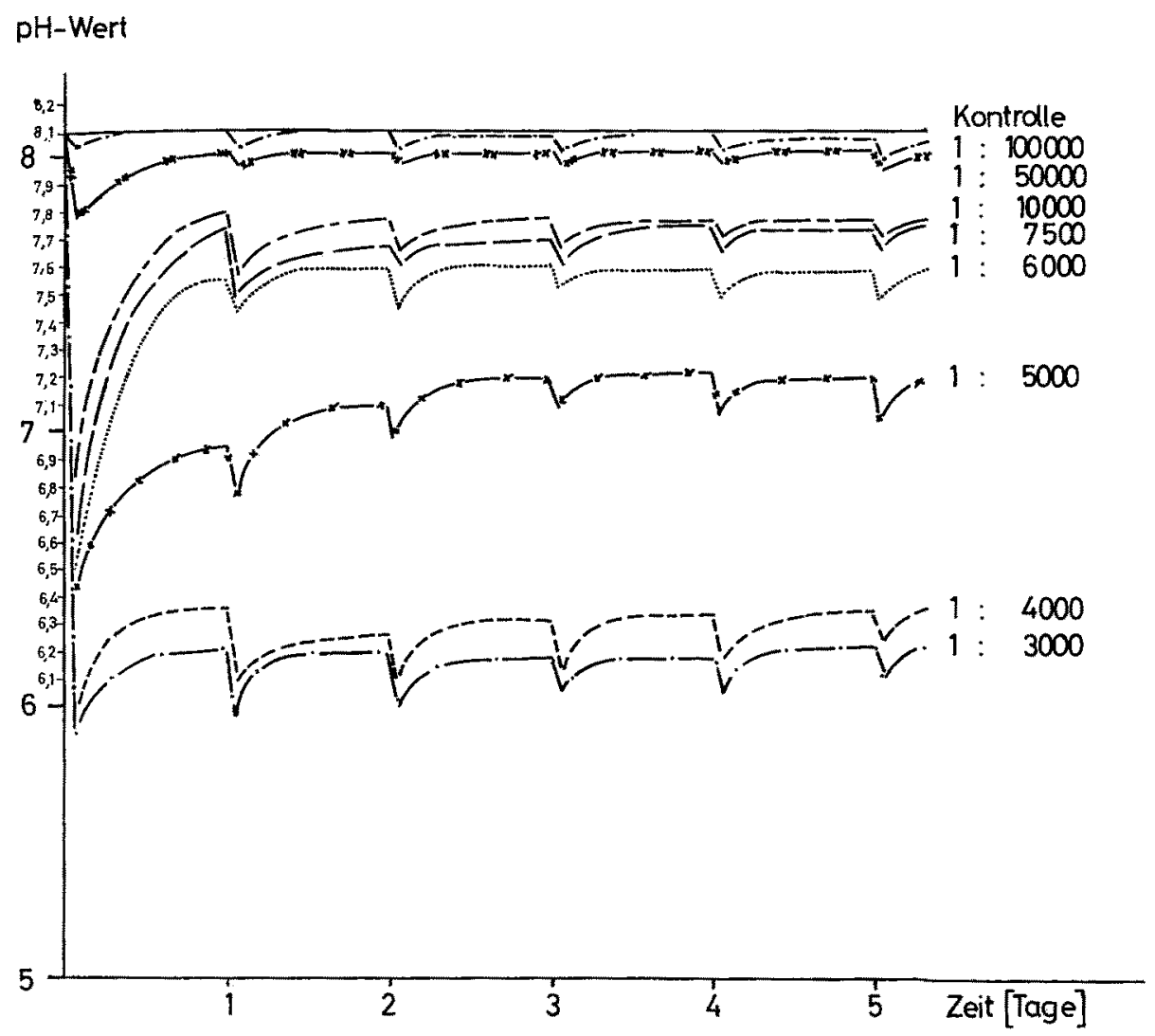

Abb. 9: Prorocentrum micans. Verlauf der pH-Anderung im "Chemostat mit konstantem Volumen" bei täglichem Zusatz von frischem Abwasser-Seewassergemisch

ca. $200 \mathrm{~cm}^{3}$ Versuchslösung ausgetauscht. Abbildung 8 zeigt den Verlauf der Wachstumskurven der Populationen über einen Zeitraum von 25 Tagen. Wie in dem vorstehend geschilderten Versuch verläuft die Kurve der Zelldichte im Kontrollversuch als horizontale Gerade bei 1000 Zellen $/ \mathrm{cm}^{3}$. In der Verdünnung von $1: 100000$ liegt die Zelldichte bis zum 6 . Tage ebenfalls annähernd bei $1000 \mathrm{Zellen} / \mathrm{cm}^{3}$ und sinkt bis zum Versuchsende langsam auf $800 \mathrm{Zellen} / \mathrm{cm}^{3} \mathrm{ab}$. In den Konzentrationen 1:50000 und 1:32000 fällt die Zelldichte gleich in den ersten 3 Tagen auf $900 \mathrm{Zellen} / \mathrm{cm}^{3} \mathrm{ab}$, hält sich bis zum 12. Tage etwa bei $800 \mathrm{Zellen} / \mathrm{cm}^{3}$ und sinkt bis zum 25. Tage langsam auf 
$700 \mathrm{bzw}$. 550 Zellen/ $\mathrm{cm}^{3}$ weiter ab. Die Verdünnung $1: 25000$ bleibt bis zum 8 . Tage etwa bei 800 Zellen $/ \mathrm{cm}^{3}$, sinkt bis zum 15. Tage relativ schnell bis auf 470 Zellen ab und bleibt bis Versuchsende etwa bei diesem Wert stehen ( $420 \mathrm{Zellen} / \mathrm{cm}^{3}$ ). In den Konzentrationen $1: 10000$ und $1: 7500$ ist der Abfall der Kurven vom 8. Tage an ebenfalls beschleunigt und führt bei Versuchsende zu Zelldichten von 200 bzw. 140 Zellen/ $\mathrm{cm}^{3}$. Der Kurvenverlauf der Konzentrationen 1:5000, 1:4000 und 1:3000 hebt sich deutlich von der Schar der vorigen Kurven ab. In den ersten 2 Tagen fällt die Zelldichte sofort auf Werte von $700 \mathrm{bzw}$. $650 \mathrm{Zellen} / \mathrm{cm}^{3} \mathrm{ab}$. In allen 3 Stufen zeigt sich in den nächsten Tagen jedoch eine deutliche Erholungsphase bis zum 6. Tage (1:5000 und $1: 4000)$ bzw. 4. Tage $(1: 3000)$. Dann aber kommt die Vermehrung vollends zum Stillstand, so daß die Kurven relativ parallel bis auf Werte von 26 (1:5000), 21 $(1: 4000)$ und $8(1: 3000)$ Zellen $/ \mathrm{cm}^{3}$ bei Versuchsende absinken. Vom 7. bzw. 5. Tage an verlaufen sie daher parallel zur theoretischen Verdünnungskurve.

In Abbildung 9 sind die zugehörigen Werte der $\mathrm{pH}-$ Kontrollen in den Versuchsgefäßen vor und nach dem täglichen Austausch an Versuchslösung aufgetragen. Die Kontrolle hält den $\mathrm{pH}$-Wert von 8,1 nahezu konstant äber die ganze Versuchsdauer ein. Wie im vorigen Versuch gibt die erstmalige Abwasserzugabe den stärksten $\mathrm{pH}$ Abfall. An den folgenden Tagen ist der $\mathrm{pH}$-Abfall entsprechend geringer, da nur $1 / 5$ des Chemostat-Volumens durch frisches $\mathrm{Abwassergemisch}$ ausgetauscht wird.

In der Konzentration von $1: 100000$ weicht der pH-Wert täglich nur um 0,06 vom Grundwert von 8,1 ab. Bei einem Gemisch von 1:50000 sinkt der pH-Wert am ersten Tage auf 7,8 ab. Der Grundwert liegt in den folgenden Tagen bei 8,02. Die tägliche $\mathrm{pH}-\mathrm{Ab}$ weichung beträgt wiederum nur 0,06 . In Konzentrationen von 1:10000 bis $1: 6000$ liegen die Grundwerte bei $7,8 \mathrm{bzw}, 7,6$, die täglichen Spitzenwerte bei 7,7 bzw. 7,5. Die pH-Kurve der Konzentration $1: 5000$ hebt sich deutlich von den vorigen ab. Nach einer Anfangsspitze von $\mathrm{pH} \mathrm{6,4}$ am ersten Tage steigt der Grundwert im Laufe von 3 Tagen auf $\mathrm{pH} 7,2$ an. Die tägliche pH-Schwankung beträgt etwa 0,14 vom Grundwert. Bei Konzentrationen von $1: 4000$ und $1: 3000$ ist ein weiterer Sprung im Kurvenbild abzulesen. Die pH-Werte stellen sich nach anfänglichem Absinken auf 5,95 bzw. 5,90 auf Grundwerte von ca. 6,3 bzw. 6,2 ein. Die täglichen Spitzenwerte liegen um 6,15 bzw. 6,05. Die tägliche pH-Änderung liegt daher im Mittel bei 0,15.

\section{Ceratium furca}

\section{Versuche in Schreiberlösung}

Die Ergebnisse der Kulturexperimente zur Ermittlung der Vermehrungsrate sind in Abbildung 10 dargestellt. Ceratium furca wächst bei $18^{\circ} \mathrm{C}$ und 8000 Lux (Tageslicht-Leuchtröhren) in $300 \mathrm{~cm}^{3}$ Schreiberlösung von einer Zelldichte von $2 \mathrm{Zellen} / 10 \mathrm{~cm}^{3}$ innerhalb von 34 Tagen auf 5000 Zellen $/ 10 \mathrm{~cm}^{3}$ heran. Aus dem Anstieg der Geraden berechnet sich eine mittlere Teilungsgeschwindigkeit von je einer Zellteilung in drei Tagen.

Zusätzlich wurde in Abbildung 10 noch das Ergebnis eines Kulturversuchs mit Ceratium horridum abgebildet. Unter gleichen Bedingungen steigt die Zellzahl inner- 
halb von 22 Tagen bis zu einer Dichte von $100 \mathrm{Zellen} / 10 \mathrm{~cm}^{3}$. Die Teilungsgeschwindigkeit liegt bei einer Zellteilung in etwa 4 Tagen.

\section{Versuche unter Abwassereinfluß}

Nach der auf p. 25 unter Punkt (1) beschriebenen Methode wurden die Testorganismen in ein um 24 Stunden gealtertes $\mathrm{Abwasser-Seewasser-Gemisch} \mathrm{eingesetzt}$ und über einen Zeitraum von 18 Tagen weiterkultiviert. Die Ergebnisse der täglichen Zellzählungen im Kontrollversuch und in 6 verschiedenen Abwasserkonzentrationen sind in Abbildung 11 dargestellt. Die Kulturen wurden mit einer Zelldichte von $10 \mathrm{Zel}-$

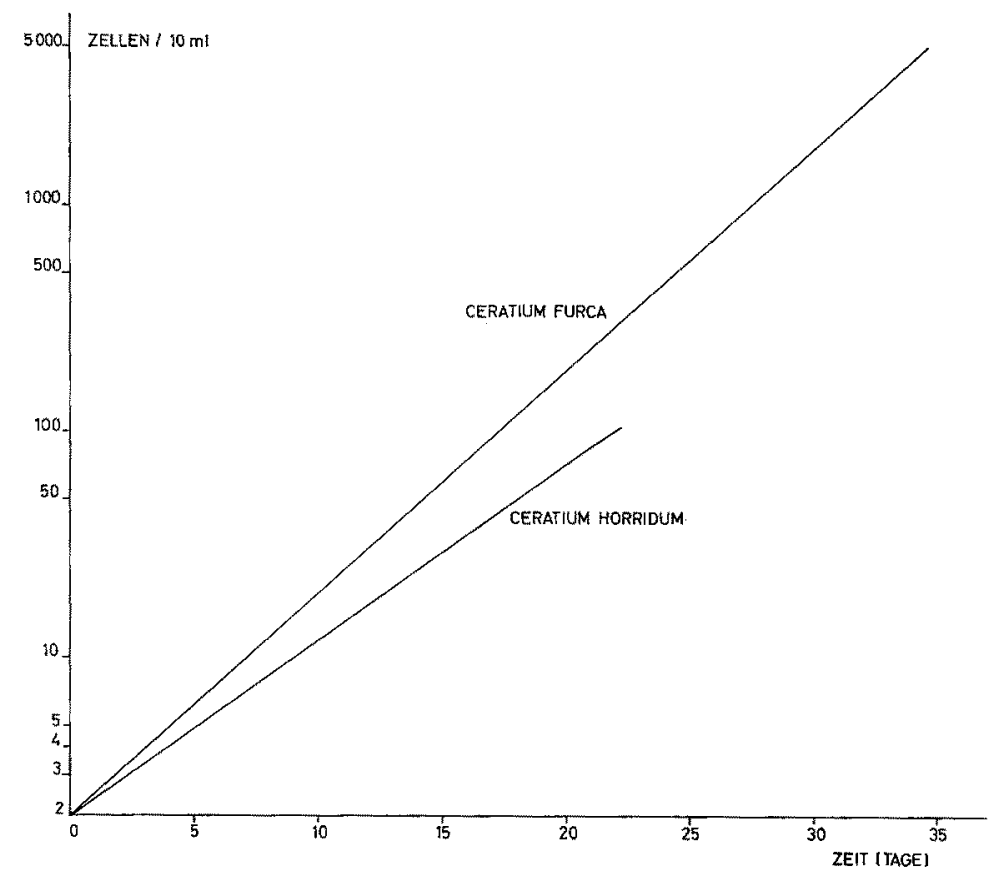

Abb. 10: Ceratium furca. Zunahme der Zelldichte bei $18^{\circ} \mathrm{C}$ in $300 \mathrm{~cm}^{3}$ Schreiberlösung bei 8000 Lux (Tageslichtleuchtröhren)

len $/ \mathrm{cm}^{3}$ angesetzt. In der Kontrolle steigt die Zelldichte innerhalb der Versuchszeit auf $185 \mathrm{Zellen} / \mathrm{cm}^{3}$ an. In der Abwasserkonzentration 1:100 000 macht sich bereits ein leichtes Absinken der Vermehrungsrate bemerkbar. Die Kultur wächst im gleichen Zeitraum nur noch auf die Dichte von $130 \mathrm{Zellen} / \mathrm{cm}^{3}$. In der Konzentration 1:50 000 ist die Beeinträchtigung der Vermehrung bereits deutlich ausgeprägt, da die Zelldichte bei Versuchsende nur noch $63 \mathrm{Zellen} / \mathrm{cm}^{3}$ erreicht. In den folgenden Konzentrationsstufen von $1: 10000$ bis $1: 4000$ liegen die Zelldichten bei Versuchsende nur noch bei 50 bis $22 \mathrm{Zellen} / \mathrm{cm}^{3}$. Bei einer Konzentration von 1:3500 stirbt in den ersten 14 Tagen ein großer Teil der Zellen ab. Die Überlebenden halten sich bis zum Verșuchșende etwa auf gleicher Zelldichte von 2 Zellen $/ \mathrm{cm}^{3}$, 


\section{DISKUSSION}

Die Ergebnisse der Versuche unter Abwassereinfluß sind im Rahmen dieser Arbeit von besonderem Interesse. Die Methodik dieser Versuche wurde jedoch erst aus den vorher durchgeführten Experimenten zur Ermittlung optimaler Kulturbedingungen

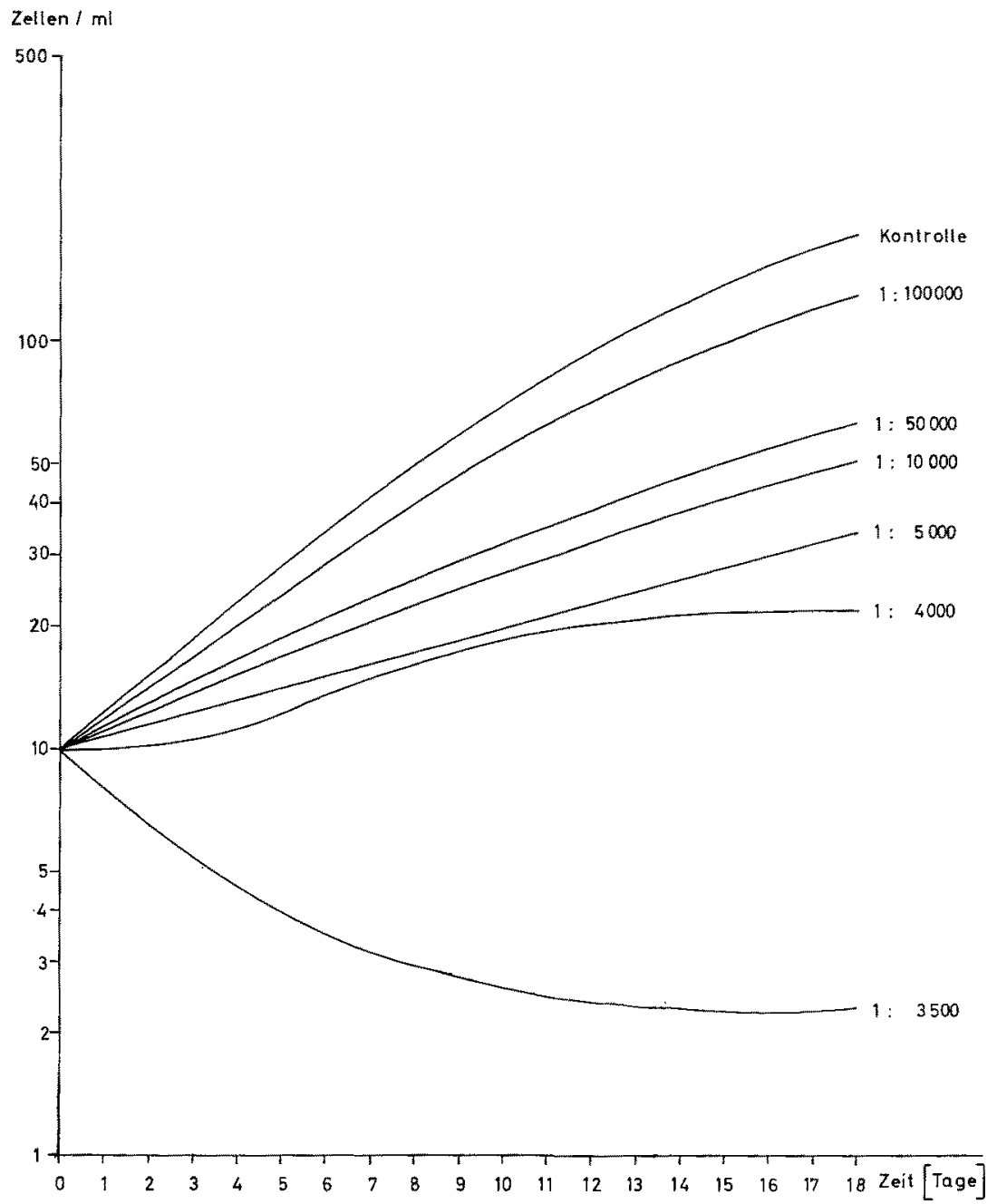

Abb. 11: Ceratium furca. Verlauf der Wachstumskurven bei Einwirkung von um 24 Stunden gealterten Abwasser-Seewassergemischen in $200 \mathrm{~cm}^{3}$ Lösung

heraus entwickelt. Hierfür wurden die Algen sowohl in geschlossener Flasche bei konstantem Volumen (Abb. 1 und 10) als auch im Chemostat-Verfahren (Abb. 2) gezïchtet. Ein Vergleich der nach beiden Methoden gewonnenen Ergebnisse zeigt übereinstimmende Vermehrungsraten in der exponentiellen Wachstumsphase der Populationen. 
Bei der in begrenztem Volumen folgenden Phase des Absterbens der Kultur ist das Phänomen der Erholung auf Grund von Remineralisierungsvorgängen aus abgestorbenen Individuen bei $18^{\circ} \mathrm{C}$ deutlich ausgeprägt ( $\mathrm{Abb}$. 1). Für die Beurteilung von $\mathrm{Ab}$ wassereinflüssen wurde in den späteren Versuchen aber immer nur auf die Beeinflussung in der exponentiellen Phase Bezug genommen; sie schließt die durch das begrenzte Versuchsvolumen bedingten Erschöpfungserscheinungen der Kulturen noch nicht ein und entspricht daher den Verhältnissen in freier Natur. Auch im Chemostat-Verfahren befindet sich die Kultur in der exponentiellen Phase der Vermehrung. Die Ergebnisse der nach beiden Methoden durchgeführten Abwasserversuche sind daher direkt miteinander vergleichbar.

Das Kriterium zur Beurteilung der Toxizität des geprüften Abwassers bildete die Beeinflussung der Vermehrungsrate. HalsBand (1968) unterscheidet bezüglich des schädigenden Einflusses von Abwässern drei Phasen: (a) Letalitätsgrenze (die Organismen sind nicht lebensfähig und gehen nach kurzer Zeit ein); (b) Störungsschwelle (die Organismen unterliegen stärkeren physiologischen Schwankungen, die jedoch noch nicht unmittelbar zum Tode führen; können die Organismen in biologisch gesundere Zonen abwandern, so überleben sie, gelingt ihnen dies nicht, führen die Schädigungen auf die Dauer gesehen zum 'Tode); (c) Erträglichkeitsgrenze (geringe Schädigungen können von den Organismen kompensiert werden). HaLsBands Klassifizierung bezieht sich auf die Reaktion von Einzelorganismen. Bei der Deutung vorliegender Versuche muß man sich jedoch vor Augen halten, daß die Ergebnisse das Verhalten ganzer Populationen wiedergeben. Ein Rückgang der Vermehrungsrate einer Art führt in freier Natur bereits zu einer Störung des dynamischen Gleichgewichts der Biocoenose.

Die kritische Grenze, die einer Beurteilung von Abwassereinleitungen zugrunde gelegt werden muß, liegt bereits bei einer ersten, deutlich erkennbaren Beeinflussung der Vermehrungsrate. Der Grenzwert bleibt dabei noch innerhalb der von HalsBand definierten „Erträglichkeitsgrenze“, da die Schädigungen von den Testorganismen bis zu einem gewissen Grad kompensiert werden können. Die "Störungsschwelle" geht unmittelbar in die "Letalitätsgrenze" über, weil sich die Testorganismen über die ganze Versuchsdauer unter Abwassereinfluß befanden und nicht in sauberes Wasser ausweichen konnten.

Die Ergebnisse aller Versuche zeigen übereinstimmend, daß jegliche Abwasserzugabe bereits einen Einfluß auf die Vermehrungsrate erkennen läßt. Bei Prorocentrum micans bleibt die Schädigung in Konzentrationen von 1:100 000 und 1:50 000 noch fast unmerklich. In der Konzentration von $1: 32000$ ist die Schädigung bereits deutlich ausgeprägt. Bei Ceratium furca beginnt die Grenze einer deutlichen Schädigung bereits in der Konzentration von 1:50 000. In beiden Arten nimmt die Beeinträchtigung der Vermehrungsrate in den höheren Konzentrationen fließend zu. Bei Prorocentrum micans überwiegt im Mischungsverhältnis 1:5000 in den ersten Tagen bereits die Zahl der absterbenden Organismen die Zahl der zuwachsenden. Die Letalitätsgrenze liegt bei Konzentrationen unterhalb von $1: 2500$. Für Ceratium furca liegt die Letalitätsgrenze bereits unterhalb der Konzentration $1: 3500$.

Bei Versuchsbeginn macht sich die Schädigung jeweils am stärksten bemerkbar. Es folgt eine Phase der Erholung. Die Organismen besitzen bis zu einem gewissen Grad die Fähigkeit, sich an die veränderten Umweltbedingungen anzupassen. Ferner kann 
auch eine Selektion resistenter Individuen oder resistenter Entwicklungsphasen der Individuen innerhalb der Population stattfinden; sie ermöglicht die Erholungsphase und das Fortbestehen der Kulturen. In den mit frischem Abwasser beschickten Versuchen bedingt auch die erstmalige starke pH-Erniedrigung eine extreme Anfangsbelastung, die mit einer maximalen Anfangsschädigung beantwortet wird.

Ein Vergleich der in gealtertem Abwassergemisch gezüchteten Kulturen mit den Kulturen unter Einfluß von frischem $A b$ wasser zeigt keine wesentlichen Unterschiede der Ergebnisse. Auch die Versuche nach dem Chemostatprinzip kommen grundsätzlich zu gleichen Resultaten. Die Höhe der Abwasserkonzentration hat über längere Zeiträume hinweg gesehen demnach größere Bedeutung für die Reaktionen der Organismen als der Einfluß der Anteile von frischem Abwassergemisch zu gealtertem. Haben sich die Organismen erst einmal an das Milieu angepaßt, sind sie in der Lage, tägliche pHSchwankungen in gewissen Grenzen zu überstehen.

Für die Frage, welche Bestandteile des Abwassers die toxische Wirkung hauptsächlich bedingen, können drei Möglichkeiten in Betracht gezogen werden: (1) Einfluß des $\mathrm{H}_{2} \mathrm{SO}_{4}$-Anteils, (2) Einfluß des $\mathrm{Fe}(\mathrm{OH})_{3}-\mathrm{Niederschlages} \mathrm{und} \mathrm{(3)} \mathrm{Einfluß} \mathrm{toxischer} \mathrm{Me-}$ tallionen.

Die subletalen Schädigungen in Konzentrationen von 1:100000 und 1:50000 sind vermutlich nicht auf die geringen pH-Verschiebungen des Abwasser-Seewassersystems zurückzuführen, sondern durch die Wirkung des Fe-Niederschlages oder toxischer Metallionen zu erklären. Die entstehenden Fe-Hydroxidflocken wirken als Fällungsmittel für schwebende Partikel und schließen diese ein. Ein Teil der Organismen könnte auf diese Weise abgefangen werden. In höheren Konzentrationen macht sich zusätzlich die $\mathrm{pH}$-Verschiebung bemerkbar. In Konzentrationen von 1:5000 pendelt der $\mathrm{pH}$-Wert im Chemostat-Verfahren um den Wert 7 und liegt bei höheren Konzentrationen $z$ wischen 6 und 6,5. Im Verfahren mit nur einmaliger Abwasserzugabe zu Versuchsbeginn stellt sich der pH-Wert auf 7,5 (1:5000) bzw. 7,0 (1:4000) und 6,8 $(1: 2500)$ ein. Eine Klärung der Wirkungsmechanik eines so komplexen Abwassers läßt sich aber mit Sicherheit erst anhand yon Versuchen mit den einzelnen Abwasserbestandteilen erzielen und soll künttigen Versuchen vorbehalten sein.

Die Ergebnisse vorliegender Versuche entsprechen in der Größenordnung der schädigenden Konzentrationen den Untersuchungen von KINNE \& ROSENTHAL (1967) an Eiern und Larven von Clupea harengus und von KINNE \& SCHUManN (1968) an jungen Gobius pictus und Solea solea.

Der Schwerpunkt vorliegender Versuche wurde auf die Entwicklung von Kulturmethoden gelegt, die subletale Einflüsse toxischer Abwässer über lange Zeiträume erkennen lassen. Die Prüfung der Industrieabwässer der Titanwerke GmbH bot dabei den praktischen Anlaß. Es erschien angebracht, die Ergebnisse der Versuche bereits vor der ersten Einleitung der Abwässer im Seegebiet bei Helgoland zu veröffentlichen.

Ziel weiterer Untersuchungen wird es sein, Standardmethoden zu entwickeln, um mit geeigneten Organismen die Toxizität unbekannter Abwässer im Züchtungsverfahren zu testen. 


\section{ZUSAMMENFASSUNG}

1. Die geplante Einleitung von Industrieabwässern eines Titandioxidwerkes in ein Seegebiet 12 Seemeilen nordwestlich Helgolands gibt den Anlaß, Untersuchungen über die Toxizität dieser Abwässer im Labor aufzunehmen. Die Untersuchungen wurden an zwei marinen Flagellaten durchgeführt, Prorocentrum micans und Ceratium furca (Dinophyta). Beide Arten treten im Plankton der Nordsee häufig auf.

2. Grundlage der Toxizitätsuntersuchungen boten Züchtungsexperimente über den Einfluß von Temperatur, Licht und Nährstoffbedarf. Die Experimente wurden mit Unialgalkulturen sowohl in Flaschen als auch nach dem Chemostat-Prinzip durchgeführt. Die Vermehrungsrate der Algen wurde durch Auszählen definierter Proben bestimmt.

3. In den Versuchen unter Abwassereinfluß bildete die Beeinträchtigung der Vermehrungsrate das Kriterium zur Beurteilung der Toxizität. Die Testorganismen wurden in Konzentrationen von 1:100000 bis 1:2500 Teilen Abwasser: Seewasser über einen Zeitraum von mehreren Wochen kultiviert. In getrennten Versuchsansätzen wurde der Einfluß von frischen und von gealterten Abwasser-Seewassergemischen untersucht. Der Verlauf der $\mathrm{pH}$-Anderung in den Versuchsgefäßen wurde über die gesamte Versuchsdauer gemessen.

4. Die Ergebnisse lassen erkennen, daß die Vermehrungsrate der Algen bereits durch Abwasserkonzentrationen von 1:100 000 leicht beeinflußr wird. Deutlich schädigende Wirkungen treten bei Ceratium furca in Konzentrationen von $1: 50000$, bei Prorocentrum micans von 1:32000 Teilen Abwasser: Seewasser auf. In Konzentrationen von $1: 5000$ kommt bei Prorocentrum micans das Wachstum der Kulturen vollkommen zum Erliegen. Die letalen Konzentrationen liegen für Ceratium furca unterhalb 1:3500, für Prorocentrum micans unterhalb 1:2500 Teilen Abwasser: Seewasser.

5. Bei der Festsetzung der Grenzwerte, die bei Abwassereinleitungen in See eingehalten werden müßten, sollten insbesondere die Ergebnisse von Untersuchungen über subletale Schädigungen zugrunde gelegt werden. Die hier vorgelegten Untersuchungen über die Beeinflussung der Vermehrungsrate mariner Flagellaten durch Industrieabwässer leisten dazu einen Beitrag.

Danksagung. Der Deutschen Forschungsgemeinschaft wird für die finanzielle Unterstützung des Forschungsvorhabens gedankt. Meinem technischen Assistenten, Herrn R. BeNEsCH, sage ich Dank und Anerkennung für seine Hilfe bei der Durchführung dieser Arbeit. Ebenso möchte ich Fräulein P. Hosumbex für die Anfertigung der Zeichnungen danken.

\section{ZITIERTE LITERATUR}

Bernhard, M., Zattera, A. \& Filesi, P., 1966. Suitability of various substances for use in the culture of marine organisms. Pubbl. Staz. zool. Napoli 35, 89-104.

BraArud, T., 1961. Cultivation of marine organisms as a means of understanding environmental influences on populations. In: Oceanography. Ed. by M. Sears. Publ. Am. Ass. Adomt. Sci. 67, 271-298. 
FogG, G. E., 1965. Algal cultures and phytoplankton ecology. The Athlone Press, London, $126 \mathrm{pp}$.

HalsBand, E., 1968. Physiologische Untersuchungsmethoden zur Bestimmung des Schädlichkeitsgrades von Abwassergiften in Süß-, Brack- und Salzwasser. Helgoländer wiss. Meeresunters. 17, 224-246.

Hueck, H. J. \& Adema, D. M. M., 1968. Toxicological investigations in an artifical ecosystem. A progress report on copper toxicity towards algae and daphniae. Helgoländer teiss. Meeresunters. 17, 188-199.

Kinne, O. \& Rosenthal, H., 1967. Effects of sulfuric water pollutants on fertilization, embryonic development and larvae of the herring, Clupea barengus. Mar. Biol. 1, 65-83.

- \& SchumanN, K. H., 1968. Biologische Konsequenzen schwefelsäure- und eisensulfathaltiger Industrieabwässer. Mortalität junger Gobius pictus und Solea solea (Pisces). Helgoländer wiss. Meeresunters. 17, 141-155.

Stosch, H. A. v. \& Drebes, G., 1964. Entwicklungsgeschichtliche Untersuchungen an zentrischen Diatomeen. IV. Die Planktondiatomee Stepbanopyxis turris - ihre Behandlung und Entwicklungsgeschichte. Helgoländer wiss. Meeresunters. 11, 209-257.

Anschrift des Autors: Dr. H. KAYseR

Biologische Anstalt Helgoland

Meeresstation

2192 Helgoland 